\title{
Fresh perspectives on an established technique: Pulsed Amplitude Modulation (PAM) chlorophyll a fluorescence
}

\author{
Guanqiang Zuo ${ }^{1}$, Robert Aiken ${ }^{1}$, Naijie Feng ${ }^{1}$, Dianfeng Zheng ${ }^{1}$, Haidong Zhao ${ }^{1}$, Thomas \\ Avenson ${ }^{1}$, and Xiaomao $\operatorname{Lin}^{2}$
}

${ }^{1}$ Affiliation not available

${ }^{2}$ Kansas State University

June 8, 2021

\begin{abstract}
Pulsed amplitude modulation (PAM) chlorophyll a fluorescence provides information about photosynthetic energy transduction. When reliably measured, chlorophyll a fluorescence provides detailed information about critical in vivo photosynthetic processes. Such information has recently provided novel and critical insights into how yield potential of crops could be improved. While PAM chlorophyll a fluorometers measure fluorescence intensity per se, herein we articulate the criteria by which instrumentally detected intensities can be assumed to assess fluorescence yield, a phenomenon quite different than fluorescence intensity and one that provides critical insight about how solar energy is variably partitioned into the biosphere. An integrated mathematical, phenomenological, and practical discussion of many useful chlorophyll a fluorescence parameters is presented. We draw attention to, and provide examples of, potential uncertainties that can result from incorrect methodological practices and potentially problematic instrumental design features. Fundamentals of fluorescence measurements are discussed, including the major assumptions underlying the signals and the methodological caveats about taking measurements during both dark- and lightadapted conditions. Key fluorescence parameters are discussed in the context of recent applications under environmental stress. Nuanced information that can be gleaned from intracomparisons of fluorescence-derived parameters and intercomparisons of fluorescence-derived parameters with those based on other techniques is elucidated.
\end{abstract}

\section{Hosted file}

Avenson and Lin main text submission.docx available at https://authorea.com/users/418565/ articles/525272-fresh-perspectives-on-an-established-technique-pulsed-amplitudemodulation-pam-chlorophyll-a-fluorescence 